\title{
Renormalization Group in Non-Relativistic Quantum Statistics
}

\author{
Juha Honkonen ${ }^{1, \star}$, M. V. Komarova ${ }^{2}$, Yu. G. Molotkov ${ }^{2}$, M. Yu. Nalimov ${ }^{2}$, and \\ Yu. A. Zhavoronkov² \\ ${ }^{1}$ Department of Military Technology, National Defence University, \\ Santahaminantie 2, 00860 Helsinki, Finland \\ ${ }^{2}$ Department of Theoretical Physics, Faculty of Physics, Saint Petersburg State University, \\ Ul'yanovskaya 1, Petrodvorets, 198504 Saint Petersburg, Russia
}

\begin{abstract}
Dynamic behaviour of a boson gas near the condensation transition in the symmetric phase is analyzed with the use of an effective large-scale model derived from time-dependent Green functions at finite temperature. A renormalization-group analysis shows that the scaling exponents of critical dynamics of the effective multi-charge model coincide with those of the standard model A. The departure of this result from the description of the superfluid transition by either model $\mathrm{E}$ or $\mathrm{F}$ of the standard phenomenological stochastic models is corroborated by the analysis of a generalization of model $\mathrm{F}$, which takes into account the effect of compressible fluid velocity. It is also shown that, contrary to the single-charge model A, there are several correction exponents in the effective model, which are calculated at the leading order of the $\epsilon=4-d$ expansion.
\end{abstract}

\section{Introduction}

The analysis of the critical dynamics of the superfluid transition has a long history. The attempts to derive critical scaling behaviour with the aid of microscopic models [1] and phenomenological stochastic models [2,3] have given different results. Rather quickly, the models E and F in the standard classification [4] became preferred to the Matsubara formalism used in the microscopic approach $[2,5]$. However, an unambiguous result for the dynamic critical exponent at the physically relevant value of the expansion parameter $\epsilon$ has been elusive in the stochastic setup [6,7].

The model of bosons with pair interactions [8] has been used to describe the superfluid transition and it is also the basis of the analysis of the Bose-Einstein condensation [9]. We have re-examined the microscopic approach to the problem of the phase transition in the boson system with the use of time-dependent Green functions at finite temperature (GF@FT) instead of the Matsubara functions used in the previous attempts. Moreover, we have used the functional representation [10] of Green functions which allows to derive an effective large-scale model for interacting bosons and demonstrate its renormalizability by standard power counting [11]. We have carried out a field-theoretic renormalization-group ( $\mathrm{RG}$ ) analysis of the effective model and obtained results which differ from those of both the early microscopic approach and models E and F. In particular, it is shown that the critical dimension of the order parameter $\eta$ and the dynamic exponent $z$ coincide with those of the

^e-mail: juha.honkonen@helsinki.fi 
standard model A [4, 6]. Critical exponents describing corrections to scaling are shown to be different from those of the model A. The effective model possesses three charges, contrary to the model A which has a single charge. Therefore, in the effective model there are three correction exponents determined by the eigenvalues of the Jacobi matrix of the RG $\beta$ functions. All these correction exponents have been calculated at the leading order of the $\epsilon=4-d$ expansion.

\section{Real-time Green functions at finite temperature}

We are investigating a non-relativistic gas of scalar bosons with local repulsive density-density interaction near the critical point of condensation. Thus, the Hamilton operator is chosen in the form

$$
\hat{H}=\int d x\left[\hat{\psi}^{+}\left(-\frac{\hbar^{2} \nabla^{2}}{2 m}-\mu\right) \hat{\psi}+\frac{g}{2} \hat{\psi}^{+2} \hat{\psi}^{2}\right]
$$

To study long-time asymptotics we use the GF@FT in the setup of Keldysh [12], in which the initial correlations are neglected and the averaging is carried out in the grand-canonical ensemble of the free system. Of the four bare propagators of perturbation theory generated by the Hamilton operator (1) and the grand-canonical averaging two are purely oscillatory in time (see, e.g. [13]), which brings about unusual divergences in individual Feynman diagrams [14]. To regularize these divergences it is convenient to introduce the attenuation factor $\exp \left[-\gamma\left|t-t^{\prime}\right|\right](\gamma>0)$ in all bare two-point functions [11]. Physically, this regularization corresponds to dissipation due to interaction, technically it allows to write unambiguously the generating function of GF@FT as a functional integral:

$$
\begin{array}{r}
G\left(A, A^{+}\right)=\int \mathcal{D} \psi_{2} \mathcal{D} \psi_{2}^{+} \mathcal{D} \psi_{1} \mathcal{D} \psi_{1}^{+} \exp \left(A^{+} \psi_{2}+A \psi_{2}^{+}\right. \\
+2 \psi_{1}^{+} \gamma[1+\bar{n}(\boldsymbol{k})] \psi_{2}+2 \psi_{2}^{+} \gamma \bar{n}(\boldsymbol{k}) \psi_{1}+\psi_{1}^{+}\left\{\partial_{t}+i \omega(\boldsymbol{k})-\gamma[1+2 \bar{n}(\boldsymbol{k})]\right\} \psi_{1} \\
\left.-\psi_{2}^{+}\left\{\partial_{t}+i \omega(\boldsymbol{k})+\gamma[1+2 \bar{n}(\boldsymbol{k})]\right\} \psi_{2}+\frac{i g}{2 \hbar}\left(\psi_{1}^{+2} \psi_{1}^{2}-\psi_{2}^{+2} \psi_{2}^{2}\right)\right),
\end{array}
$$

where $\omega(\boldsymbol{k})=\epsilon(\boldsymbol{k}) / \hbar=\left(\hbar^{2} \boldsymbol{k}^{2} / 2 m-\mu\right) / \hbar$ and $\bar{n}(\boldsymbol{k})$ is the mean occupation number of the state with $\boldsymbol{k}$ in the free boson gas: $\bar{n}(\boldsymbol{k})=\{\exp [\beta \epsilon(\boldsymbol{k})]-1\}^{-1}$. In (2) the subscript 2 refers to the physical fields and 1 to the auxiliary fields. All integrals are implied in the shorthand notation in (2). Time integrals are taken over the whole real time axis.

To carry out a renormalization-group analysis it is necessary to use a different set of fields [11] given by the relations $\eta=\left(\psi_{2}-\psi_{1}\right) / \sqrt{2}, \xi=\left(\psi_{2}+\psi_{1}\right) / \sqrt{2}$ and their conjugates. In terms of these fields the action part in the exponential (2) assumes the form

$$
\begin{gathered}
\frac{i}{\hbar} S\left(\eta, \eta^{+}, \xi, \xi^{+}\right)=\eta^{+}\left[-\partial_{t}-i \omega(\boldsymbol{k})-\gamma\right] \xi+\xi^{+}\left[-\partial_{t}-i \omega(\boldsymbol{k})+\gamma\right] \eta \\
-2 \eta^{+}[(1+2 \bar{n}) \gamma] \eta-\frac{i g}{2 \hbar}\left(\eta^{+} \xi^{+} \xi^{2}+\xi^{+2} \xi \eta+\xi^{+} \eta^{+} \eta^{2}+\eta^{+2} \eta \xi\right) .
\end{gathered}
$$

For the RG analysis we have constructed an effective large-scale model with definite canonical dimensions of the fields. In the critical region $p^{2} \sim \mu \rightarrow 0$ the mean occupation number $\bar{n}(\boldsymbol{p})$ factor is singular and the leading contribution of the unregularized Keldysh function

$$
\Delta_{\mathrm{IR}}^{K}(\omega, \boldsymbol{k})=\frac{4 \pi T_{C} \delta[\omega-\omega(\boldsymbol{k})]}{\hbar \omega(\boldsymbol{k})},
$$

where $T_{C}$ is the critical temperature in energy units, is the necessary generalized homogeneous function, which - together with the retarded and advanced propagators brought about by the free action (3) - leads to canonical dimensions of the effective infra-red (IR) model $d_{\xi}=d_{\xi^{+}}=d / 2-1$, 
$d_{\eta}=d_{\eta^{+}}=d / 2+1$ [11]. Thus, the critical dimension is $d_{c}=4$ and the two rightmost vertex structures in (3) are IR irrelevant and discarded. To keep these canonical dimensions in the regularized model it is necessary to choose the attenuation parameter $\gamma \propto k^{2}$.

\section{Renormalization-group analysis of the effective model}

We use the massless scheme to calculate renormalization constants [10] putting $\mu=0$, so that $\bar{n}(\boldsymbol{k}) \approx 2 m T_{C} / \hbar^{2} k^{2}$. The extra parameters $\hbar, m$ and $T_{C}$ are removed from the action by the scaling of coordinates, time and fields. In the streamlined notation the basic action of the effective IR model is

$$
\begin{array}{r}
\tilde{S}=-4 \alpha \eta^{+} \eta+\eta^{+}\left[-\partial_{t}+\nabla^{2}(u \alpha i-\alpha)\right] \xi+\xi^{+}\left[-\partial_{t}+\nabla^{2}(u \alpha i+\alpha)\right] \eta \\
-\frac{i \alpha g}{2} \eta^{+} \xi^{+} \xi^{2}-\frac{i \alpha \bar{g}}{2} \xi^{+2} \eta \xi
\end{array}
$$

corresponding to the weight $\exp (\tilde{S})$ in (2). For convenience, the coupling constants are expressed in the form $\alpha g$ and $\alpha \bar{g}$ with the real coefficient $\alpha$ of the attenuation factor. The real parameter $u$ is a nonperturbative charge typical of models in dynamic critical phenomena [10].

Action (4) is similar to that brought about by the stochastic Gross-Pitaevskii equation [15], although the content of the fields is quite different: in [15] the counterpart of $\xi$ is the order parameter field and the counterpart of $\eta$ is the auxiliary response field of the stochastic setup. In (4) both $\xi$ and $\eta$ are linear combinations of the physical field $\psi_{2}$ and the auxiliary field $\psi_{1}$ of the Keldysh formalism. It should also be noted that the stochastic setup of the boson problem is based on a solution of the evolution equation for the density operator in which several approximations are used [16]. In the Keldysh approach the initial correlations are neglected but otherwise the quantum dynamics is exact and the effective model similar to that of the stochastic approach is a result of the standard renormalization procedure.

The basic action (4) gives rise to propagators $\left(\Delta_{\eta \eta^{+}}(t, \boldsymbol{k})=0\right)$

$$
\begin{aligned}
\Delta_{\xi \xi^{+}}(t, \boldsymbol{k}) & =\frac{2}{k^{2}} \exp \left[-i \alpha u k^{2}\left(t-t^{\prime}\right)-\alpha k^{2}\left|t-t^{\prime}\right|\right], \\
\Delta_{\xi \eta^{+}}(t, \boldsymbol{k}) & =\theta\left(t-t^{\prime}\right) \exp \left[-i \alpha u k^{2}\left(t-t^{\prime}\right)-\alpha k^{2}\left|t-t^{\prime}\right|\right], \\
\Delta_{\eta \xi^{+}}(t, \boldsymbol{k}) & =-\theta\left(t^{\prime}-t\right) \exp \left[-i \alpha u k^{2}\left(t-t^{\prime}\right)-\alpha k^{2}\left|t-t^{\prime}\right|\right] .
\end{aligned}
$$

The structure of the interaction in (3) is such that the closed loops of the temporal step functions of the propagators (5) make certain classes of Feynman diagrams to vanish [11]. In particular, oneirreducible diagrams with external $\xi$ or $\xi^{+}$arguments only vanish identically. Moreover, the basic action (4) obeys the the symmetry $\tilde{S}\left(\eta^{+}, \eta, \xi^{+}, \xi, g, \bar{g}\right)=\tilde{S}^{*}\left(-\eta^{+},-\eta, \xi^{+}, \xi, \bar{g}^{*}, g^{*}\right)$ (integration by parts is implied). The subsequent restrictions on renormalization render the effective IR model multiplicatively renormalizable with the renormalized action

$$
\tilde{S}=-Z_{0} \eta \eta^{+}+\eta^{+}\left(-Z_{1} \partial_{t}+Z_{2} \nabla^{2}\right) \xi+\xi^{+}\left(-Z_{3} \partial_{t}+Z_{4} \nabla^{2}\right) \eta-Z_{5} \eta^{+} \xi^{+} \xi \xi-Z_{6} \eta \xi \xi^{+} \xi^{+} .
$$

The basic action (4) is invariant under the constant gauge transformation $\xi \rightarrow \xi t^{i c}, \xi^{+} \rightarrow \xi^{+} t^{-i c}$, $\eta \rightarrow \eta t^{i c}, \eta^{+} \rightarrow \eta^{+} t^{-i c}$ as well. We use this invariance to prescribe real renormalization constants to the fields $\eta$ and $\eta^{+}$.

We seek the solution for renormalization group functions in the physical subspace $\bar{g}=g^{*}$ and parametrize the coupling constants by real $g_{1}$ and $g_{2}$ as $g=g_{1}+i g_{2}$ and $\bar{g}=g_{1}-i g_{2}$. Counterterms 
in the leading order have been calculated in [11]. In the MS scheme they give rise to (see, e.g. [10]) the $\beta$ functions of charges and $\gamma$ functions of fields and the parameter $\alpha$ in the form

$$
\begin{aligned}
& \beta_{g 1}=-\frac{2\left(2 u^{2}+3\right) g_{1} g_{2}+u g_{2}^{2}-u g_{1}^{2}}{8 \pi^{2}\left(u^{2}+1\right)}-\epsilon g_{1}, \\
& \beta_{g 2}=\frac{2 u g_{1} g_{2}-\left(4 u^{2}+5\right) g_{2}^{2}+g_{1}^{2}}{8 \pi^{2}\left(u^{2}+1\right)}-\epsilon g_{2}, \\
& \beta_{u}=\frac{\left(u^{2}+6\right) u g_{2}^{2}-u g_{1}^{2}+6 g_{1} g_{2}}{64 \pi^{4}\left(u^{2}+9\right)} \\
& -\frac{\left(u^{2}+1\right)}{128 \pi^{4}}\left[\left(g_{1}^{2}+g_{2}^{2}\right) \operatorname{Im} M_{2}+2\left(g_{1}^{2}-g_{2}^{2}\right) \operatorname{Im} M_{3}+4 g_{1} g_{2} \operatorname{Re} M_{3}\right] . \\
& \gamma_{\alpha}=-\frac{3 g_{1}^{2}+2 u g_{1} g_{2}-\left(2 u^{2}+9\right) g_{2}^{2}}{128 \pi^{4}\left(u^{2}+9\right)}+\frac{\left(g_{1}^{2}+g_{2}^{2}\right)}{128 \pi^{4}}\left(\operatorname{Re} M_{2}-u \operatorname{Im} M_{2}\right) \\
& +\frac{\left(g_{1}^{2}-2 u g_{1} g_{2}-g_{2}^{2}\right) \operatorname{Re} M_{3}}{64 \pi^{4}}-\frac{\left(u g_{1}^{2}+2 g_{1} g_{2}-u g_{2}^{2}\right) \operatorname{Im} M_{3}}{64 \pi^{4}}, \\
& \gamma_{\eta}=\frac{3 g_{1}^{2}+2 u g_{1} g_{2}-\left(2 u^{2}+9\right) g_{2}^{2}}{256 \pi^{4}\left(u^{2}+9\right)}-\frac{\left(g_{1}^{2}+g_{2}^{2}\right)}{256 \pi^{4}}\left(M_{1}+\operatorname{Re} M_{2}-u \operatorname{Im} M_{2}\right) \\
& -\frac{\left(g_{1}^{2}-2 u g_{1} g_{2}-g_{2}^{2}\right) \operatorname{Re} M_{3}}{128 \pi^{4}}+\frac{\left(u g_{1}^{2}+2 g_{1} g_{2}-u g_{2}^{2}\right) \operatorname{Im} M_{3}}{128 \pi^{4}} \\
& \gamma_{\xi}=-\frac{3 g_{1}^{2}+2 u g_{1} g_{2}-\left(2 u^{2}+9\right) g_{2}^{2}}{256 \pi^{4}\left(u^{2}+9\right)}-\frac{\left[g_{1}^{2}+2(u+2 i) g_{1} g_{2}-g_{2}^{2}\right] \operatorname{Re} M_{3}}{128 \pi^{4}} \\
& -\frac{\left(g_{1}^{2}+g_{2}^{2}\right)\left[\operatorname{Re} M_{2}+(u+2 i) \operatorname{Im} M_{2}-M_{1}\right]}{256 \pi^{4}} \\
& -\frac{\left[(u+2 i) g_{1}^{2}-2 g_{1} g_{2}-(u+2 i) g_{2}^{2}\right] \operatorname{Im} M_{3}}{128 \pi^{4}},
\end{aligned}
$$

where

$$
\begin{gathered}
M_{1}=\frac{A+3 B-\pi u-2 u \arctan C_{-}}{u^{2}+1}, \quad M_{2}=\frac{(u-i)^{2}\left(2 i \arctan \left(2 C_{+}\right)-\pi i-A-B\right)}{\left(u^{2}+1\right)^{2}}, \\
M_{3}=\frac{(u+i)^{2}(B-2 i \arctan (u / 3))}{\left(u^{2}+1\right)^{2}}, \quad A=\ln \left(u^{2}+1\right), B=\ln \left(\frac{u^{2}+9}{16}\right), C_{ \pm}=\frac{u^{2} \pm 3}{4 u} .
\end{gathered}
$$

In contrast with the models $\mathrm{E}$ and $\mathrm{F}$, in which the large number of nontrivial nodes of $\beta$ functions hinders the choice of the IR-stable fixed point, the $\beta$ functions (6) vanish simultaneously in only three cases:

(i) The trivial fixed point $g_{1 *}=g_{2 *}=0$ with arbitrary $u_{*}$. Eigenvalues of the Jacobi matrix $\omega_{i j}=\partial_{i} \beta_{j}$ are $(-\epsilon,-\epsilon, 0)$ confirming the IR instability of the fixed point.

(ii) $g_{1 *}=0, g_{2 *}=-8 \pi^{2} \epsilon / 5, u_{*}=0$. Eigenvalues of the $\omega$ matrix are $\left(\epsilon, \epsilon / 5,2 \log (4 / 3) \epsilon^{2} / 25\right)$. The positive sign of all eigenvalues at $\epsilon>0$ means that this fixed point is IR stable.

(iii) $g_{2 *}=-2 \pi^{2} \epsilon, w_{*} \equiv 1 / u_{*}=0$ with arbitrary $g_{1 *}$. The infinite value $u_{*}$ is taken into account due to the nonperturbative nature of the charge $u$. This fixed point leads to eigenvalues of the matrix $\omega_{i j}$ equal to $(\epsilon,-\epsilon / 16,0)$. Thus, this is an unstable saddle point. It corresponds to quantummechanical behaviour of the system described by oscillating propagators without attenuation. 
The calculation of the $\gamma$ functions at the IR stable fixed point (ii) yields the critical dimensions:

$$
\gamma_{\xi}^{*}=\frac{\epsilon^{2}}{100}, \quad \gamma_{\eta}^{*}=\left(12 \log \frac{4}{3}-1\right) \frac{\epsilon^{2}}{100}, \quad \gamma_{\alpha}^{*}=\left(1-6 \log \frac{4}{3}\right) \frac{\epsilon^{2}}{50} .
$$

It should be noted that at the only IR-stable fixed point (ii) the charges $g$ and $\bar{g}$ are purely imaginary. Moreover, in terms of variables $\phi$ and $\phi^{\prime}$ defined by relations $\phi_{1}^{\prime}=\eta^{+}-\eta, \phi_{2}^{\prime}=i\left(\eta^{+}+\eta\right), \phi_{1}=\left(\xi^{+}+\xi\right) / 2$, $\phi_{2}=i\left(\xi^{+}-\xi\right) / 2$ the action (4) at the fixed point (ii) assumes the form

$$
\tilde{S}_{*}=\alpha \phi^{\prime 2}+\phi^{\prime}\left[-\partial_{t} \phi+\alpha\left(\nabla^{2} \phi+\frac{g_{2 *}}{2} \phi^{3}\right)\right]
$$

which coincides with the De Dominicis-Janssen action $[17,18]$ of the stochastic two-component model A [10] up to notation. The negative sign of $g_{2 *}$ in (8) matches the correct sign in model A [10]. This is why it is not surprising that the result (7) coincides with the leading order of the $\epsilon$ expansion for the critical dimensions in model A.

\section{Stochastic model with density fluctuations}

The results of the microscopic approach are supported by the generalization of the F model $[2,3]$ to incorporate the effect of fluctuating fluid velocity $\boldsymbol{v}$. The consistent stochastic problem taking into account mode coupling theory [10] for the complex order parameter $\psi$, field of temperature fluctuations $m$ and the velocity $\boldsymbol{v}$ is

$$
\begin{aligned}
\partial_{t} \psi & =-v_{i} \partial_{i} \psi+\lambda(1+i b)\left(\nabla^{2} \psi-\frac{g_{1} \psi^{+} \psi^{2}}{3}+g_{2} m \psi\right) \\
& +i \lambda g_{3} \psi\left(g_{2} \psi^{+} \psi-m-r w \frac{v^{2}}{2}\right)+f_{\psi^{+}}, \\
\partial_{t} m & =-\left(\frac{r^{2} c^{2} w}{\rho_{0}}+1\right) \partial_{i}\left(m v_{i}\right)-\lambda u \nabla^{2}\left(g_{2} \psi^{+} \psi-m-r w \frac{v^{2}}{2}\right) \\
& +i \lambda g_{3}\left(\psi^{+} \nabla^{2} \psi-\psi \nabla^{2} \psi^{+}\right)+f_{m}, \\
\partial_{t} v_{i} & =\frac{\gamma}{\rho_{0}} \nabla^{2} v_{i}+\frac{[\zeta d+(d-2) \gamma] \partial_{j} \partial_{i} v_{i}}{\rho_{0} d}-\partial_{j}\left(v_{i} v_{j}\right) \\
& -\frac{1}{w \rho_{0}}\left(\partial_{i} \psi^{+}\right)\left(\nabla^{2} \psi-\frac{g_{1} \psi^{+} \psi^{2}}{3}+g_{2} m \psi\right)-\frac{1}{2} \partial_{i} v^{2} \\
& -\frac{1}{w \rho_{0}}\left(\partial_{i} \psi\right)\left(\nabla^{2} \psi^{+}-\frac{g_{1} \psi^{+2} \psi}{3}+g_{2} m \psi^{+}\right) \\
& +\left(\frac{r c^{2}}{\rho_{0}}+\frac{1}{r w}\right) \partial_{i}\left(g_{2} \psi^{+} \psi-m-\frac{w r v^{2}}{2}\right)+f_{v},
\end{aligned}
$$

where $\gamma$ and $\zeta$ are the shear and bulk viscosity, respectively, $\lambda$ is the kinetic coefficient, $b$ and $g_{3}$ are the mode-coupling coefficients, $u$ is the nonperturbative charge of the model F, $c$ is the speed of sound and $\rho_{0}$ the mean density. It is assumed that $\rho=\rho_{0}+m$. For simplicity, some dimensional parameters are put equal to the unity. The uncorrelated random sources $f_{i}$ are Gaussian with zero mean. 
The standard procedure [10] leads to the dynamic action with auxiliary fields $\psi^{\prime}, \psi^{+\prime}, m^{\prime}, v^{\prime}$ :

$$
\begin{aligned}
S_{\mathrm{dyn}} & =b_{1} \psi^{+\prime} \psi^{\prime}-b_{2} m^{\prime} \partial^{2} m^{\prime}+b_{3} \partial_{i} v_{j}^{\prime} \partial_{i} v_{j}^{\prime}+b_{4} \partial_{j} v_{j}^{\prime} \partial_{i} v_{i}^{\prime}+a_{1} \psi^{+\prime} \nabla^{2} \psi \\
& +a_{2} \psi^{\prime} \nabla^{2} \psi^{+}+a_{3} m^{\prime} \partial_{i} v_{i}+a_{4} m^{\prime} \nabla^{2} m+a_{5} v_{i}^{\prime} \nabla^{2} v_{i}+a_{6} v_{i}^{\prime} \partial_{i} \partial_{j} v_{j} \\
& +a_{7} v_{i}^{\prime} \partial_{i} m-\psi^{+\prime} \partial_{t} \psi-\psi^{\prime} \partial_{t} \psi^{+}-m^{\prime} \partial_{t} m-v^{\prime} \partial_{t} v+\psi^{+\prime}\left(-v_{i} \partial_{i} \psi\right. \\
& \left.+a_{8} \psi^{+} \psi \psi+a_{9} m \psi+a_{10} \psi v^{2}\right)+\psi^{\prime}\left[-v_{i} \partial_{i} \psi^{+}+a_{11}\left(\psi^{+}\right)^{2} \psi\right. \\
& \left.+a_{12} m \psi^{+}+a_{13} \psi^{+} v^{2}\right]+m^{\prime}\left[-m \partial_{i} v_{i}+a_{14} \nabla^{2}\left(\psi^{+} \psi\right)+a_{15} \nabla^{2} v^{2}\right. \\
& \left.+a_{16} \psi^{+} \nabla^{2} \psi+a_{17} \psi \nabla^{2} \psi^{+}\right]+v_{i}^{\prime}\left[-\partial_{j}\left(v_{i} v_{j}\right)+a_{18}\left(\partial_{i} \psi^{+}\right) \nabla^{2} \psi\right. \\
& +a_{19} \psi^{+} \psi^{2} \partial_{i} \psi^{+}+a_{20} m \psi \partial_{i} \psi^{+}+a_{21}\left(\partial_{i} \psi\right) \nabla^{2} \psi^{+}+a_{22}\left(\psi^{+}\right)^{2} \psi \partial_{i} \psi \\
& \left.+a_{23} m \psi^{+} \partial_{i} \psi+a_{24} \partial_{i}\left(\psi^{+} \psi\right)+a_{25} \partial_{i} v^{2}\right] .
\end{aligned}
$$

The analysis of the canonical dimensions reveals that $a_{3}=-r^{2} c^{2} w / \rho_{0}-1$ is the most IR-relevant parameter. The leading IR-relevant propagators homogeneous under scaling $\omega, \boldsymbol{k} \rightarrow s^{2} \omega, s \boldsymbol{k}$ are

$$
\begin{array}{r}
\Delta_{m m}(\omega, \boldsymbol{k})=\frac{b_{34}}{a_{7}^{2}}, \quad \Delta_{m^{\prime} m}(\omega, \boldsymbol{k})=\frac{a_{56} k^{2}+i \omega}{a_{3} a_{7} k^{2}}, \quad \Delta_{m^{\prime} v_{\|}}(\omega, \boldsymbol{k})=-\frac{i \vec{k}}{a_{3} k^{2}}, \\
\delta_{v_{\|}^{\prime} m}(\omega, \boldsymbol{k})=-\frac{i \vec{k}}{a_{7} k^{2}}, \quad \Delta_{v_{\|} m}(\omega, \boldsymbol{k})=\frac{a_{3} b_{34}\left(i \omega-a_{4} k^{2}\right)+a_{7} b_{2}\left(a_{56} k^{2}+i \omega\right)}{a_{3}^{2} a_{7}^{2} k^{2}} i \vec{k}, \\
\Delta_{v_{\| v_{\|}}}(\omega, \boldsymbol{k})=\frac{b_{2}}{a_{3} a_{7} k^{2}} P_{\|}, \quad \Delta_{v_{\|}^{\prime} v_{\|}}(\omega, \boldsymbol{k})=\frac{a_{4} k^{2}+i \omega}{a_{3} a_{7} k^{2}} P_{\|},
\end{array}
$$

where $P_{\|}$is the longitudinal projection operator. In the numerator of propagator $\Delta_{\vartheta_{\|} m}$, the product $a_{3} b_{34}$ appears, in which $b_{34}=[\zeta+(2 d-2) \gamma / d] / \rho_{0}$ is irrelevant. The product, however, has the canonical dimension equal to zero and is thus marginal.

It is even more important that the propagators $\Delta_{m^{\prime} m}, \Delta_{m^{\prime} v_{\|}}, \Delta_{v_{\|} m}, \Delta_{v_{\|} \|_{\|}}$and $\Delta_{v_{\|}^{\prime} v_{\|}}$contain the IRrelevant coefficient $a_{3}$ in the denominator. It renders irrelevant the contributions of all the diagrams in which these propagators are inserted. Analogously, all the contributions of the propagators $\Delta_{v_{\perp} v_{\perp}}$ and $\Delta_{m m}$ may be discarded, because they have the irrelevant factor $b_{34}$ in the numerator. It is important to note that the irrelevant propagators may be discarded, because at vertices of the action (9) there are no charges with positive canonical dimensions.

All propagators with the field $m^{\prime}$ are irrelevant so that vertices with $m^{\prime}$ do not contribute to leading IR asymptotics of Green functions. Inverting the matrix of the rest marginal propagators $\Delta_{\psi^{\prime} \psi_{+}}, \Delta_{\psi \psi^{\prime}+}$, $\Delta_{\psi \psi+}, \Delta_{v_{\|}^{\prime} m}, \Delta_{v_{\perp}^{\prime} \nu_{\perp}}$ and discarding irrelevant vertices of action (9) we arrive at the generating function for the order parameter

$$
G_{\mathrm{eff}}\left(A, A^{+}\right)=\int \mathcal{D} \psi^{+} \mathcal{D} \psi \mathcal{D} \psi^{\prime+} \mathcal{D} \psi^{\prime} \mathcal{D} m \mathcal{D} m^{\prime} \mathcal{D} v \mathcal{D} v^{\prime} \delta\left(m^{\prime}\right) \exp \left(S_{\text {dyn }}^{\prime}+A \psi^{+}+A^{+} \psi\right),
$$

where $\delta\left(m^{\prime}\right)$ is the functional $\delta$ function and the effective action is

$$
\begin{aligned}
S_{\mathrm{dyn}}^{\prime} & =b_{1} \psi^{+\prime} \psi^{\prime}+a_{1} \psi^{+\prime} \nabla^{2} \psi+a_{2} \psi^{\prime} \nabla^{2} \psi^{+} \\
& +a_{5} v_{\perp}^{\prime} \nabla^{2} v_{\perp}+a_{7} v_{i}^{\prime} \partial_{i} m-\psi^{+\prime} \partial_{t} \psi-\psi^{\prime} \partial_{t} \psi^{+}-v_{\perp}^{\prime} \partial_{t} v_{\perp} \\
& +\psi^{+\prime}\left[-v_{i} \partial_{i}(\psi)+a_{8} \psi^{+} \psi \psi+a_{9} m \psi+a_{10} \psi v^{2}\right] \\
& +\psi^{\prime}\left[-v_{i} \partial_{i} \psi^{+}+a_{11} \psi^{+} \psi \psi^{+}+a_{12} m \psi^{+}+a_{13} \psi^{+} v^{2}\right] \\
& +m^{\prime}\left[-m \partial_{i} v_{i}+a_{14} \nabla^{2}\left(\psi^{+} \psi\right)+a_{16} \psi^{+} \nabla^{2} \psi+a_{17} \psi \nabla^{2} \psi^{+}\right] \\
& +v_{i}^{\prime}\left[-\partial_{j}\left(v_{i} v_{j}\right)+a_{24} \partial_{i}\left(\psi^{+} \psi\right)+a_{25} \partial_{i} v^{2}\right]
\end{aligned}
$$


The fields $v_{\perp}^{\prime}, v_{\|}^{\prime}$ in (11) play the role of Lagrange coefficients. They may be integrated explicitly in (10) giving rise to new functional $\delta$ functions

$$
\begin{array}{r}
\delta\left(\partial_{t} v_{\perp}+P_{\perp}(v \nabla v)-v \nabla^{2} v_{\perp}\right), \\
\delta\left(a_{7} \nabla m-P_{\|}(v \nabla v)+a_{24} \nabla\left(\psi^{+} \psi\right)+a_{25} \nabla v^{2}\right) .
\end{array}
$$

The condition imposed in (12) is the Navier-Stokes equation with the equilibrium solution $v \equiv 0$. The second condition (13) then yields $m=-a_{24}\left(\psi^{+} \psi\right) / a_{7}$ in the set of falling off fields considered in (10). Using the functional $\delta$ functions to perform integrations over $m, m^{\prime}$ and $v$ in (10) we obtain the final effective IR dynamic action describing the critical dynamics of the order parameter:

$$
\begin{array}{r}
S_{\text {eff }}=b_{1} \psi^{+\prime} \psi^{\prime}+a_{1} \psi^{+\prime} \nabla^{2} \psi+a_{2} \psi^{\prime} \nabla^{2} \psi^{+}-\psi^{+\prime} \partial_{t} \psi-\psi^{\prime} \partial_{t} \psi^{+} \\
+\psi^{+\prime}\left[a_{8} \psi^{+} \psi^{2}-a_{9} a_{24}\left(\psi^{+} \psi^{2}\right) / a_{7}\right]+\psi^{\prime}\left[a_{11}\left(\psi^{+}\right)^{2} \psi-a_{12} a_{24}\left(\psi^{+}\right)^{2} \psi / a_{7}\right] .
\end{array}
$$

It is readily seen that this action differs from the action (8) by scaling of fields and parameters only thus confirming the conclusion based on the microscopic approach.

\section{Conclusion}

In conclusion, we have shown that the critical exponents of dynamics of the superfluid transition and the Bose-Einstein condensation transition coincide with those of the two-component model A. In particular, the dynamic exponent $z$ of the transition is known to order $\epsilon^{4}$ [19]:

$$
z=2+0.014522 \epsilon^{2}+0.011059 \epsilon^{3}-0.005265 \epsilon^{4}
$$

High-order asymptotics have been studied in [20]. The most accurate results for the exponent $z$ correspond to the Borel resummation of four orders of the $\epsilon$ expansion in [21]: $z(\epsilon=1)=2.014_{-0.00}^{+011}$. Moreover, the correction exponent has been inferred at the leading order of the $\epsilon$ expansion in the standard fashion from the stability exponents of the corresponding fixed point. At the physical value of the parameter $\epsilon=1$ the smallest stability exponent - which is the exponent of corrections to critical scaling - is given by

$$
\omega=\frac{2 \epsilon^{2}}{25} \log \frac{4}{3}
$$

This value coincides with the result for the correction exponent $\eta-\eta_{c}$ of the stochastic Gross-Pitaevskii model [15] obtained by a different approach.

\section{Acknowledgments}

The work was supported by the Academy of Finland (Grant No. 325408).

\section{References}

[1] M. Suzuki, G. Igarashi, Phys. Lett. A 47, 361 (1974)

[2] B.I. Halperin, P.C. Hohenberg, E.D. Siggia, Phys. Rev. Lett. 32, 1289 (1974)

[3] B.I. Halperin, P.C. Hohenberg, E.D. Siggia, Phys. Rev. B 13, 1299 (1976)

[4] P.C. Hohenberg, B.I. Halperin, Rev. Mod. Phys. 49, 435 (1977)

[5] C. De Dominicis, E. Brézin, J. Zinn-Justin, Phys. Rev. B 12, 4945 (1975)

[6] R. Folk, G. Moser, J. Phys. A 39, R207 (2006) 
[7] M. Dančo, M. Hnatič, M.V. Komarova, T. Lučivjanský, M.Y. Nalimov, Phys. Rev. E 93, 012109 (2016)

[8] E.M. Lifshitz, L.P. Pitaevskii, Statistical Physics. Part 2: Theory of the Condensed State (Pergamon, Oxford, 1980)

[9] C.J. Pethick, H. Smith, Bose-Einstein Condensation in Dilute Gases (Cambridge University Press, Cambridge, 2002)

[10] A.N. Vasil'ev, The Field Theoretic Renormalization Group in Critical Behavior Theory and Stochastic Dynamics (Chapman Hall/CRC, Boca Raton, 2004)

[11] J. Honkonen, M.V. Komarova, Y.G. Molotkov, M.Y. Nalimov, Nucl. Phys. B 939, 105 (2019)

[12] L.V. Keldysh, Sov. Phys. JETP 20, 1018 (1965)

[13] E.M. Lifshitz, L.P. Pitaevskii, Physical Kinetics (Pergamon, Oxford, 1981)

[14] N.P. Landsman, C.G. van Weert, Phys. Rep. 145, 141 (1987)

[15] U.C. Täuber, S. Diehl, Phys. Rev. X 4, 021010 (2014)

[16] C.W. Gardiner, P. Zoller, Phys. Rev. A 55, 2902 (1997)

[17] C. De Dominicis, J. Phys. Colloq. 37, C1 (1976)

[18] H.K. Janssen, Z. Phys. B 23, 377 (1976)

[19] L.T. Adzhemyan, E.V. Ivanova, M.V. Kompaniets, S. Y. Vorobyeva, J. Phys. A 51, 155003 (2018)

[20] J. Honkonen, M.V. Komarova, M.Y. Nalimov, Nucl. Phys. B 707, 493 (2005)

[21] M.Y. Nalimov, V.A. Sergeev, L. Sladkoff, Theor. Math. Phys. 159, 496 (2009) 